\title{
La maestra de educación infantil en sus primeros años de trabajo: de la formación inicial al ejercicio de la profesión
}

\author{
The Early Childhood Teacher in Their First Years \\ of Work: From Initial Training to Exercising the \\ Profession
}

\section{María Soledad Robinson Seisdedos ${ }^{1}$, José Tejada Fernández ${ }^{2}$, y Sílvia Blanch Gelabert ${ }^{2}$}

${ }^{1}$ Universidad de Valparaíso, Chile.

${ }^{2}$ Universitat Autònoma de Barcelona, España.

\section{Resumen}

\begin{abstract}
En el proceso de construcción de la identidad profesional de la maestra de educación infantil incide la dimensión ejercicio profesional en sus primeros años de trabajo, que afecta no solo al proceso de construcción identitaria, sino también a su inserción laboral. El artículo expone las complejidades a las que se enfrentan las maestras de educación infantil en sus primeras experiencias laborales. Se trata de una investigación en la que el método utilizado es un estudio de caso múltiple, con características biográficas narrativas, aplicado a nueve educadoras en educación infantil tituladas de la misma universidad. Los resultados evidencian las siguientes complejidades: las necesidades de apoyo de la educadora principiante, que se transforman en un factor clave para su inserción; los aprendizajes significativos que contribuyen en su desarrollo profesional; las condiciones laborales que influyen de manera directa en el quehacer pedagógico, y las situaciones laborales complejas vividas con actores de la comunidad educativa. Los resultados permiten constatar que la inserción laboral está condicionada fundamentalmente por la experiencia de las educadoras principiantes, el apoyo entregado por la institución educativa de la cual forma parte, y la reflexión que realiza de este proceso que, a su vez, contribuye a la construcción de su identidad profesional.
\end{abstract}

Palabras clave: educadora principiante, identidad profesional, maestra infantil, inserción profesional, competencias profesionales

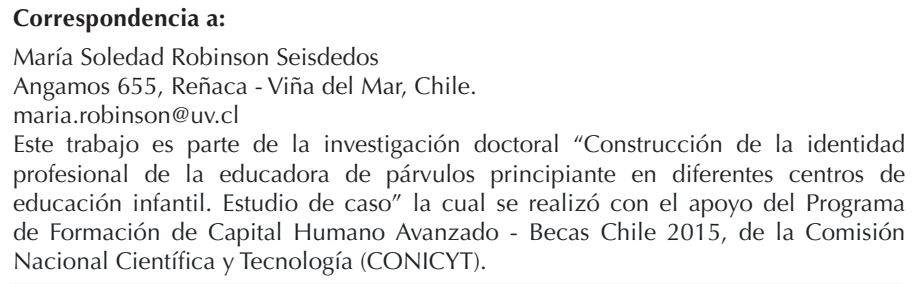

(C) 2021 PEL, http://www.pensamientoeducativo.org - http://www.pel.cl

ISSN:0719-0409ＤDI:203.262, Santiago, Chile doi: 10.7764/PEL.58.1.2021.8 


\begin{abstract}
In the process to construct the professional identity of the preschool teacher, the aspect of exercising the profession during their first few years of work affects not only the process of identity construction, but also their transition into employment. This paper outlines the complexities faced by preschool teachers in their first work experiences. The research used a multiple case study method, with narrative biographies of nine early childhood teachers from the same university in their first three years of work. The results show the following complexities: the support needed by beginning teachers, which becomes a key factor for transition into employment; the meaningful learning that contributes to their professional development; working conditions that directly influence the pedagogical work; and complex work situations experienced with actors in the educational community.
\end{abstract}

Keywords: novel educator, professional identity, early childhood teacher, transition from student to teacher, professionals' skills

\title{
Introducción
}

A lo largo del tiempo, se ha ido reconociendo que la educación infantil es una etapa crucial para facilitar los aprendizajes oportunos y pertinentes en la infancia, tanto desde una perspectiva educativa como social (Falabella, Cortázar, Godoy, y Romo, 2018). La valorización de esta etapa ha llevado a la reconceptualización de la labor de la maestra de infantil, desde un rol de tipo asistencial a uno más complejo, que incluye la función de facilitar oportunidades educativas a niños y nińas para su desarrollo y aprendizaje significativo, tanto a nivel pedagógico como a nivel socioemocional (Falabella et al., 2018).

Esta nueva mirada compleja supone que la maestra de educación infantil debe contar con conocimientos sobre el desarrollo, la entrega de aprendizajes oportunos, pertinentes y de calidad, atender a las necesidades del contexto y responder a situaciones emergentes, y poseer habilidades profesionales específicas que promuevan el aprendizaje y el bienestar de los niños y niñas. Por ejemplo, deben tener altas expectativas sobre su grupo de párvulos, ser afectivas y sensibles, cooperativas, inclusivas, y potenciar a los niños y niñas de acuerdo con sus características individuales (Peeters \& Sharmahd, 2014). A su vez, es necesario el trabajo colaborativo con diferentes agentes educativos, especialmente vinculando a las familias desde su rol como primer agente educativo (Robinson, 2019). Desde esta perspectiva, se destaca la importancia de poner al niño y la niña en el centro, y promover su desarrollo global mediante el cuidado, el juego y el desarrollo socioemocional (BubikovaMoan, Naess Hjetland, \& Wollscheid, 2019).

Sumado a lo anterior, la educadora de párvulos en Chile se enfrenta además a una carga laboral mayor que la de otros profesionales (de ocho a 11 horas diarias), siendo sus remuneraciones más bajas que las de un profesor de enseñanza básica (Viviani, 2016). Del mismo modo, debe responder a la política de resultados y rendición de cuentas que se ha instalado en los últimos ańos, tanto a nivel nacional como internacional. Prueba de ello son los estándares orientadores para las carreras de educación parvularia en Chile, en las que las futuras tituladas 
deben evidenciar al finalizar sus estudios ciertos conocimientos tanto pedagógicos como disciplinarios, lo que al trabajar, especialmente en ambientes deprivados socioeconómicamente, es difícil de llevar a la práctica. Es así como, de manera progresiva, se pone de relieve lo fundamental que es la formación profesional de las maestras de nivel infantil (Pardo y Alderstein, 2016).

En este sentido, el sistema de desarrollo profesional docente (Ley 20.903) propicia la mejora e igualdad de condiciones laborales de educadoras y profesores, lo que puede ser visto como un reconocimiento social al ejercicio profesional de la educadora de párvulos. Sin embargo, de acuerdo con lo planteado por Pardo y Alderstein (2020), los instrumentos de evaluación vinculados a la carrera docente deben ser ajustados y mejorados para que no se transformen en un proceso de evaluación pedagógica individualista, distanciado de los principios pedagógicos de la educación parvularia, el aprendizaje integral, o el trabajo con familias, entre otros. Esto podría incidir de manera directa en la identidad profesional de la educadora.

La educadora de párvulos ejerce su labor en una determinada realidad educativa, estableciendo interacciones con otros agentes educativos y comunitarios. A partir del intercambio de vivencias y opiniones con sus pares, sumado a sus experiencias previas, tanto personales como profesionales, va construyendo su identidad como educadora (Ivanova \& Skara-Mincāne, 2016). Esta identidad comienza, principalmente, en la formación inicial, mediante un proceso dinámico y relacional, en un determinado contexto y en la interacción con otros (Bolívar, 2016). De esta manera, se puede señalar que esta construcción identitaria está definida, por un lado, por las concepciones de agentes educativos y comunitarios del contexto y otros actores sociales, y, por otro, por el autoconcepto del educador en cuanto a su labor, condicionada por sus experiencias personales (Beijaard, Meijer, \& Verloop, 2004). En lo que a educación infantil se refiere, y en línea con Vergara (2014), la construcción identitaria debe sustentarse en la intencionalidad de aprendizajes oportunos, pertinentes y de calidad, lo cual no se relaciona con la representación social caracterizada por la falta de valoración del trabajo que lleva a cabo la maestra infantil (Zabalza Beraza y Zabalza Cerdeirińa, 2011).

De este modo, el educador posee características de su identidad definidas en lo profesional, laboral y personal, todas las cuales conducen a la construcción de su identidad profesional (Pelletier y Morales-Perlaza, 2018). En este sentido, la biografía y el ambiente social en el cual interactúa se vinculan (Arvaja, 2016).

A nivel profesional, la educadora infantil comparte espacio, tiempo y características con sus pares, con quienes establece un vínculo que favorece el sentido de pertenencia y un compromiso con el contexto laboral. Todos ellos son aspectos que facilitan que desarrolle su trabajo de forma más satisfactoria (Correa, Martínez-Arbelaiz, \& Fernández-Plaskoaga, 2017). Por lo tanto, la construcción identitaria facilita que la educadora encuentre un modo propio, integrando características, costumbres y formas de actuar similares a su grupo, las que a su vez son compartidas, permitiéndole de esta manera sentirse parte de una comunidad educativa (Vergara, 2014). Así, un sentido de identidad personal y profesional sólidos y compartidos, propician en los educadores principiantes la comprensión de su rol y de las demandas actuales que tienen como educadores (Chong, 2011).

La profesión de educar se caracteriza por ser variable y dinámica: las competencias que debe desarrollar la maestra infantil dependerán del escenario en el cual lleve a cabo su labor pedagógica (Perrenoud, 2004). Es en el quehacer educativo, en situaciones reales, en la respuesta o acción a realizar, donde se van desarrollando las competencias profesionales. En línea con Blanch y sus colegas (2017), uno de los aspectos determinantes en la formación de maestras en educación infantil es desarrollar las competencias y habilidades personales como la creatividad e imaginación, el pensamiento crítico, la escucha activa, la interdisciplinariedad, la resiliencia, la autonomía, el control emocional, la asertividad, la adaptabilidad, la empatía, el liderazgo, la cooperación, la responsabilidad social, y la mediación y receptividad, entre otras. 
Lo anterior se relaciona de manera directa con el trabajo a realizar no solo con niños y niñas, sino además con las familias y la comunidad educativa, comprendiendo que este nivel educativo debe responder de manera dinámica a las inquietudes, opiniones e intereses del contexto específico. De este modo, se considera el rol de la educadora como clave, ya que debe coordinar actividades y mediar pedagógicamente con niños y niñas, familias, el equipo técnico pedagógico y la comunidad (Ministerio de Educación, 2018).

Asimismo, los primeros años de trabajo como educadora se caracterizan por ser una etapa diferenciada y de transición, determinada por las tensiones y complejidades vividas en una realidad educativa desconocida (Martínez, 2012). En este contexto es donde van adquiriendo experiencias significativas y conocimientos profesionales (Marcelo y Vaillant, 2013), lo que a su vez implica una diversidad de aprendizajes, interpretando además las nuevas experiencias con los saberes adquiridos en su formación inicial (Loughran, 2014).

Por otro lado, la inserción laboral implica tensiones y conflictos al enfrentarse a situaciones nuevas y, a su vez, por la ambigüedad de su rol en el trabajo (McGillivray, 2008). En este sentido, la reflexión del quehacer pedagógico es fundamental, ya que a partir de las experiencias en la interacción con otros agentes educativos y comunitarios se van reformulando y resignificando aprendizajes, lo que va en directo beneficio de su desarrollo profesional. Junto con lo anterior, el modo de enfrentar estas tensiones y conflictos puede estar relacionado con el análisis y la reflexión sobre la práctica, así como las habilidades cognitivas desarrolladas en la formación inicial y a través del apoyo recibido en sus primeros años de trabajo (Ávalos y Sevilla, 2010; Ávalos, 2016).

Para Pillen, Den Brok y Beijaard (2013), las tensiones son vistas como conflictos internos, desde el sentir como persona y como educador, que causan cuestionamientos relacionados con sus valores. Emergen tanto de lo personal como de lo profesional, transitan entre lo que quieren y lo que es posible realizar, y también a través de los conflictos vividos, y si estos son resueltos con pares significativos o de manera autónoma (Pillen, Beijaard, \& y Den Brok, 2013).

Por otro lado, si bien se observa en los educadores principiantes una responsabilidad con el contexto social, se evidencian inseguridades vinculadas con sus capacidades y conocimientos, fundamentalmente porque carecen de experiencia en el aula. Por este motivo es que ven a sus pares que llevan más años en la institución como referentes del proceso educativo (Ávalos y Sotomayor, 2012). Las dificultades que enfrentan los educadores en sus primeros años de trabajo se vinculan con la falta de competencias, la falta de recursos y de estrategias en el manejo de situaciones, y la multiplicidad de funciones (Solís, Núńez, Contreras, Vásquez, \& Ritterhaussen, 2016).

En cuanto a las preocupaciones y desafíos que deben enfrentar las maestras de educación infantil principiantes, Sancho y sus colegas (2014) destacan entre otras las condiciones laborales inestables, la no carrera docente, las políticas educativas burocráticas y desprofesionalizantes, la distancia entre la formación inicial y la práctica, la tendencia a la repetición de modelos y prácticas, la soledad del trabajo docente, y la necesidad de seguir aprendiendo y de revisar la formación inicial y permanente de las educadoras. Si bien en este estudio se comparte casi la totalidad de las características seńaladas por los autores citados, es necesario señalar, que, en Chile, la carrera docente se establece desde la Ley 20.903, del Sistema del Desarrollo Profesional Docente, en etapa de implementación a partir del año 2017 hasta el 2026; su propósito es el reconocimiento a los profesionales de la educación frente a los logros alcanzados, propiciando, de esta manera, una mejora continua.

Otra de las dificultades con la que se enfrentan a menudo es la percepción que tienen las maestras de educación infantil de no obtener el mismo respeto que las de otras etapas superiores, viéndose así afectado el valor de su trabajo y profesión (Moloney, 2010).

El estudio realizado forma parte de una investigación que aborda el proceso de construcción de la identidad profesional en educadoras de párvulos en sus primeros años de ejercicio, teniendo presente la formación inicial y el desarrollo de las competencias. Sin embargo, dada la relevancia que posee el proceso de construcción identitaria, 
se hace necesario identificar los obstáculos que encuentran las educadoras durante los primeros años de inserción en el campo educativo. De esta forma, creemos que será posible articular de manera más eficiente las diversas iniciativas desde las políticas públicas que se han desarrollado en los últimos años para el fortalecimiento de su labor pedagógica, como innovaciones curriculares en la formación de pregrado, marco para la buena enseñanza, o estándares orientadores para las carreras de educación parvularia. Bajo esta premisa, este artículo pretende responder a la pregunta ¿Cuáles son las complejidades vinculadas a la construcción de su identidad profesional que deben enfrentar las educadoras de párvulos durante los primeros ańos de ejercicio de la profesión? El objetivo se centra en caracterizar las complejidades de la inserción laboral en las maestras principiantes en educación parvularia, identificando necesidades de apoyo en la construcción identitaria.

\section{Metodología}

\section{Diseño}

Este trabajo se sustenta en el paradigma interpretativo, que busca conocer la realidad a partir del significado que le entregan los sujetos participantes de la investigación, siendo la meta analizar las percepciones de quienes se incluyen en el estudio. El enfoque investigativo es de carácter cualitativo, al explorar y comprender el significado individual o grupal que se le atribuye a un determinado problema (Creswell, 2014). El método utilizado es un estudio de caso múltiple (Stake, 2013), ya que centra la búsqueda en un determinado fenómeno, utilizando para esto más de un caso con características compartidas, ya sea por semejanza o discrepancia.

\section{Participantes}

Para este estudio de caso múltiple se ha establecido una muestra intencional como característica y criterio de selección a nueve tituladas de la carrera de educación parvularia, de la misma universidad de la región de Valparaíso, Chile. Todas ellas se encuentran en los tres primeros años de ejercicio profesional, egresadas los años 2014 - 2015 - 2016, trabajando en centros públicos, en centros educativos particulares subvencionados y en jardines infantiles particulares. Es decir, tres por promoción, desempeñándose en su labor pedagógica con niños y niñas de nivel medio y transición.

Los criterios utilizados para seleccionar a las educadoras de párvulos tituladas de la misma universidad se basan en lo planteado por Bolívar, Fernández y Molina (2005), al señalar que uno de los aspectos que determina el proceso de adquisición de la identidad profesional es la enseñanza recibida en la formación inicial.

Por otro lado, Huberman (1989) señala que los tres primeros años de desempeño laboral hacen referencia a la entrada a la carrera profesional, conocimiento y socialización de la realidad educativa. Bajo esta misma línea, los primeros años de trabajo se caracterizan por tensiones, temores, conflictos y cuestionamientos al enfrentar situaciones desconocidas y carecer de experiencia laboral (Ávalos y Sevilla, 2010). Es en esta línea en la que se profundiza en este artículo. 
Tabla 1

Caracterización de casos

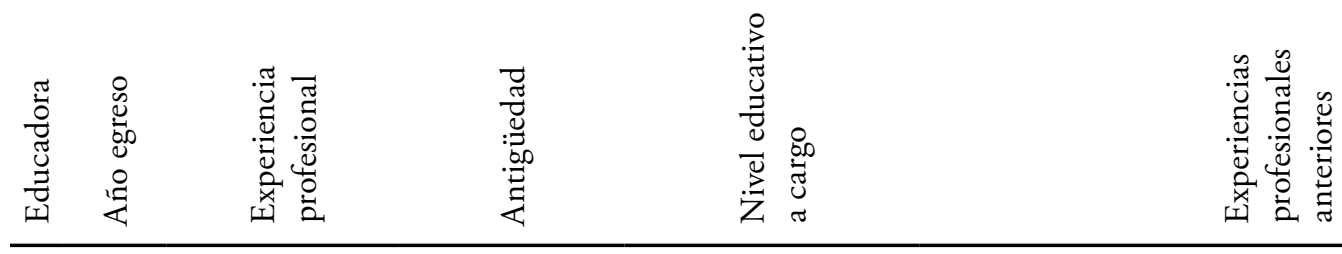

120142 años, 6 meses 1 año, 3 meses Medio mayor

Educadora de párvulos estable y de reemplazo en centros públicos de educación infantil.

220151 año, 7 meses 1 año, 4 meses $\quad 1^{\circ}$ nivel transición

Educadora de párvulos de reemplazo en centros públicos de educación infantil, 3 meses.

$32016 \quad 7$ meses $\quad 3$ meses $\quad$ Nivel medio menor

Educadora de párvulos de reemplazo en centros públicos de educación infantil, 4 meses.

420142 años, 9 meses 1 año, 7 meses $\quad 2^{\circ}$ nivel transición Educadora de párvulos en jardines infantiles particulares, 1 año, 2 meses.

\begin{tabular}{llcll}
5 & 2015 & 1 año, 10 meses & 5 meses & $1^{\circ}$ nivel transición \\
\hline 6 & $2016 \quad 9$ meses & 5 meses & $2^{\circ}$ nivel de transición
\end{tabular}

Educadora de párvulos en jardín infantil particular y en centro educativo particular subvencionado, 1 año, 5 meses.

\begin{tabular}{cccccl}
6 & 2016 & 9 meses & 5 meses & $2^{\circ}$ nivel de transición & $\begin{array}{l}\text { Educadora de párvulos de reemplazo en centros } \\
\text { públicos de educación infantil, 4 meses. }\end{array}$ \\
\hline 7 & 2014 & 7 meses & 5 meses & Nivel medio menor & $\begin{array}{l}\text { Asistente y educadora de párvulos de reemplazo, } \\
\text { directora subrogante en centro público de } \\
\text { educación infantil, 2 meses. }\end{array}$ \\
\hline 8 & 2015 & 1 año, 10 meses & 1 año, 5 meses & Heterogéneo & $\begin{array}{l}\text { Educadora de párvulos en jardín infantil } \\
\text { particular, } 5 \text { meses. }\end{array}$ \\
\hline 9 & 2016 & 10 meses & 5 meses & Nivel medio menor & $\begin{array}{l}\text { Educadora de párvulos de reemplazo centros } \\
\text { públicos de educación infantil, } 5 \text { meses. }\end{array}$ \\
\hline
\end{tabular}

Fuente: Elaboración propia.

\section{Instrumentos}

Los instrumentos utilizados corresponden a entrevistas en profundidad (nueve) y a un grupo focal, del que participaron todas las educadoras. Dadas las características del fenómeno de estudio, este trabajo considera aspectos del método biográfico narrativo, siendo aplicados relatos de vida, los cuales se refieren a narraciones autobiográficas que dan cuenta de la identidad de una persona y de la manera en la que va relatando lo vivido (Chase, 2015). Dentro de estos se distinguen los biogramas, como un mapa o esquema de su trayectoria de vida, que describen y dan cuenta de los acontecimientos relevantes que se quieren destacar. A su vez, los incidentes críticos constituyen la forma de comprender cómo un acontecimiento vivido en el pasado, ya sea positivo o negativo, cobra relevancia en el presente.

El guion de entrevista y el del grupo focal se estructuran a partir de la dimensión que se especifica en la tabla 2. De acuerdo con el carácter semiestructurado de la entrevista, es importante considerar que las preguntas son solo orientativas, por lo tanto, se podrían modificar o reformular según el desarrollo de esta, teniendo presente en todo momento el propósito que se persigue con este instrumento.

En este estudio se cuenta con categorías deductivas (aprendizajes significativos, condiciones laborales, apoyo a la educadora principiante), construidas desde el marco referencial, estableciendo una serie de pasos en la construcción de instrumentos, que van de lo general a lo específico. Las categorías inductivas, también presentes en esta investigación (situaciones laborales complejas), emergen desde los hallazgos del trabajo de campo propiamente tal. 
Tabla 2

Técnicas de recogida de información según dimensiones y categorías

Dimensión Categorías apriorísticas/ deductivas

Técnica de recogida de información

\begin{tabular}{|c|c|c|c|c|}
\hline & & Relatos de vida & Entrevista & Grupo focal \\
\hline \multirow{3}{*}{ Inserción en el campo laboral } & Aprendizajes significativos & $\mathrm{x}$ & & $\mathrm{x}$ \\
\hline & Condiciones laborales & & & $\mathrm{x}$ \\
\hline & Apoyo a la educadora principiante & & & $\mathrm{x}$ \\
\hline
\end{tabular}

Fuente: Elaboración propia.

\section{Procedimiento}

Previo a la aplicación de los instrumentos de recolección de información, los guiones de entrevistas y grupo focal fueron sometidos a juicio de expertas (Escobar-Pérez y Cuervo-Martínez, 2008). En este caso, se implicó a nueve profesionales de la educación, considerando para esto el criterio de solvencia-experticia profesional, junto con los criterios de univocidad, pertinencia e importancia.

Desde la evaluación de expertas y de acuerdo con los criterios establecidos, los resultados para la entrevista señalan mantener, sin mejorar, 13 de las 14 preguntas, y en el grupo focal se mantienen todas. A partir del pilotaje, se ha estimado un tiempo de 45 a 60 minutos para su aplicación. Para el desarrollo de la investigación, en la que las educadoras de párvulos principiantes se constituyen en los agentes clave del estudio, es necesario clarificar su participación a partir del consentimiento informado.

El procedimiento seguido fue el siguiente:

1. Se contactó a las educadoras de párvulos, una de cada contexto en estudio, dándoles a conocer el propósito de la investigación y su participación.

2. Firma de consentimiento informado.

3. Envío a través correo electrónico del instrumento "relatos de vida", en el cual debían desarrollar, en primer lugar, el biograma y, posteriormente, los incidentes críticos.

4. Realización de entrevista (previa entrega de relatos de vida desarrollados).

El análisis de la información se realiza de acuerdo con las categorías establecidas desde su semejanza/recurrencia y diferencia/relevancia (Bustingorry, Sánchez, y Ibáñez, 2006), junto con el significado otorgado al sistema de categorías deductivo-inductivo. Igualmente, en línea con Miles, Huberman y Saldaña (2014), se han establecido las tres etapas de análisis: reducción de datos, disposición de estos, e interpretación.

\section{Resultados}

En el contexto de este trabajo, como indicamos en su objetivo, se abordan las complejidades relacionadas con la dimensión de la inserción laboral. Los resultados obtenidos permiten conocer y visualizar cuáles son las situaciones vividas por las educadoras principiantes que les provocan conflicto en su quehacer pedagógico. En la tabla 3 se visualiza un resumen de las cuatro categorías y sus tipologías: deductiva o inductiva, sus frecuencias, y cuál fue el procedimiento utilizado para tal hallazgo. 
Tabla 3

Categorias y su fundamentación

\begin{tabular}{lccccccc} 
& \multicolumn{5}{c}{ Instrumento/técnica } \\
\cline { 2 - 7 } Categoría & Relatos de Vida & $\begin{array}{c}\text { Entrevistas en } \\
\text { profundidad }\end{array}$ & \multicolumn{2}{c}{ Grupo focal } \\
\cline { 2 - 8 } & F & TC & F & TC & F & TC \\
\hline Apoyo a la educadora principiante & & & 10 & E & 37 & A \\
\hline Aprendizajes significativos & 80 & A & 49 & E & 29 & A \\
\hline Condiciones laborales & 22 & E & 28 & E & 24 & A \\
\hline Situaciones laborales complejas & 28 & E & & & 15 & E \\
\hline
\end{tabular}

$F=$ Frecuencia $; C=$ Tipo de categoria $; E=$ Emergente; $A=$ Apriorística

Fuente: Elaboración propia.

En lo que respecta a las figuras 1, 2 y 3, se exponen las redes semánticas, con sus categorías y subcategorías, según el instrumento utilizado. Con relación a las categorías inductivas, se han establecido con mayúscula para diferenciarlas de las deductivas.

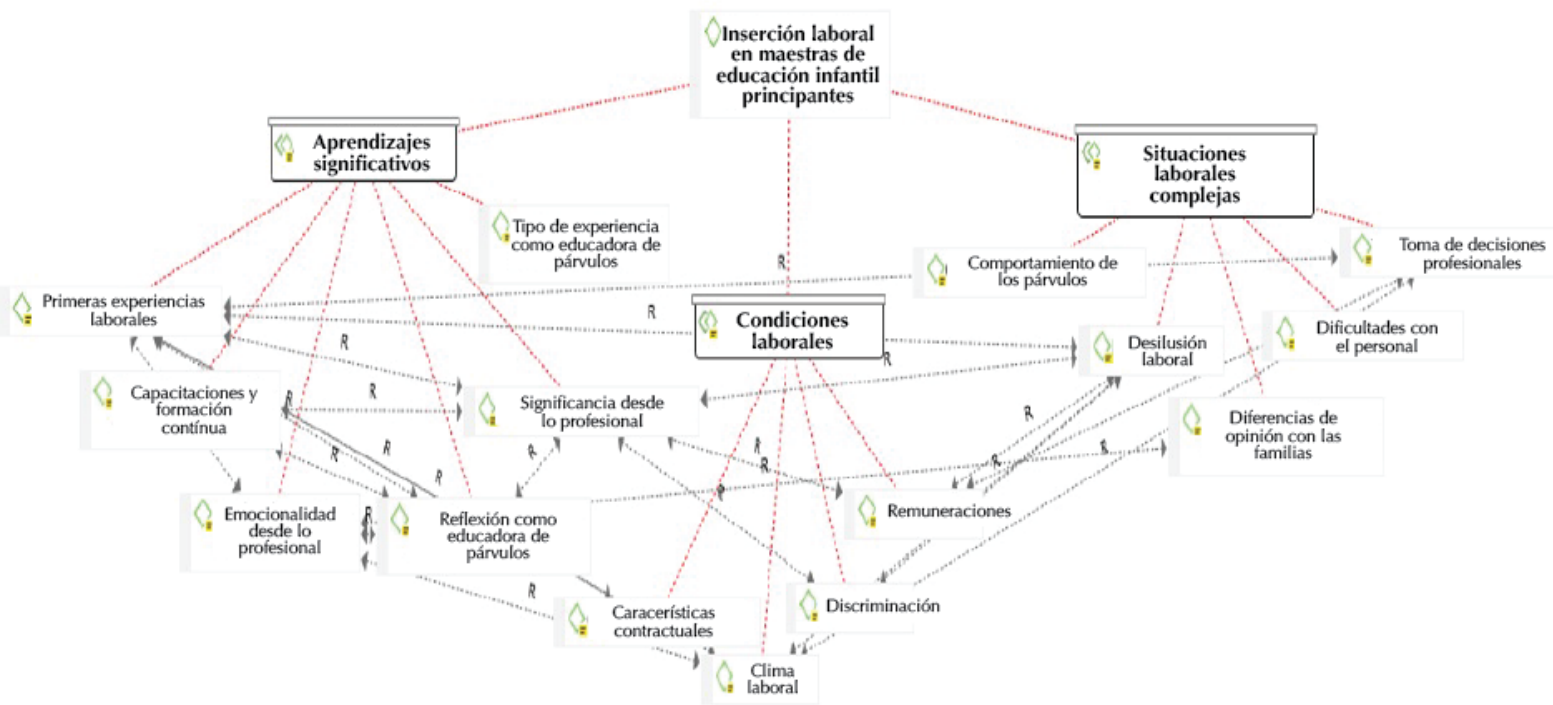

Figura 1. Red semántica relatos de vida

Fuente: Elaboración propia. 


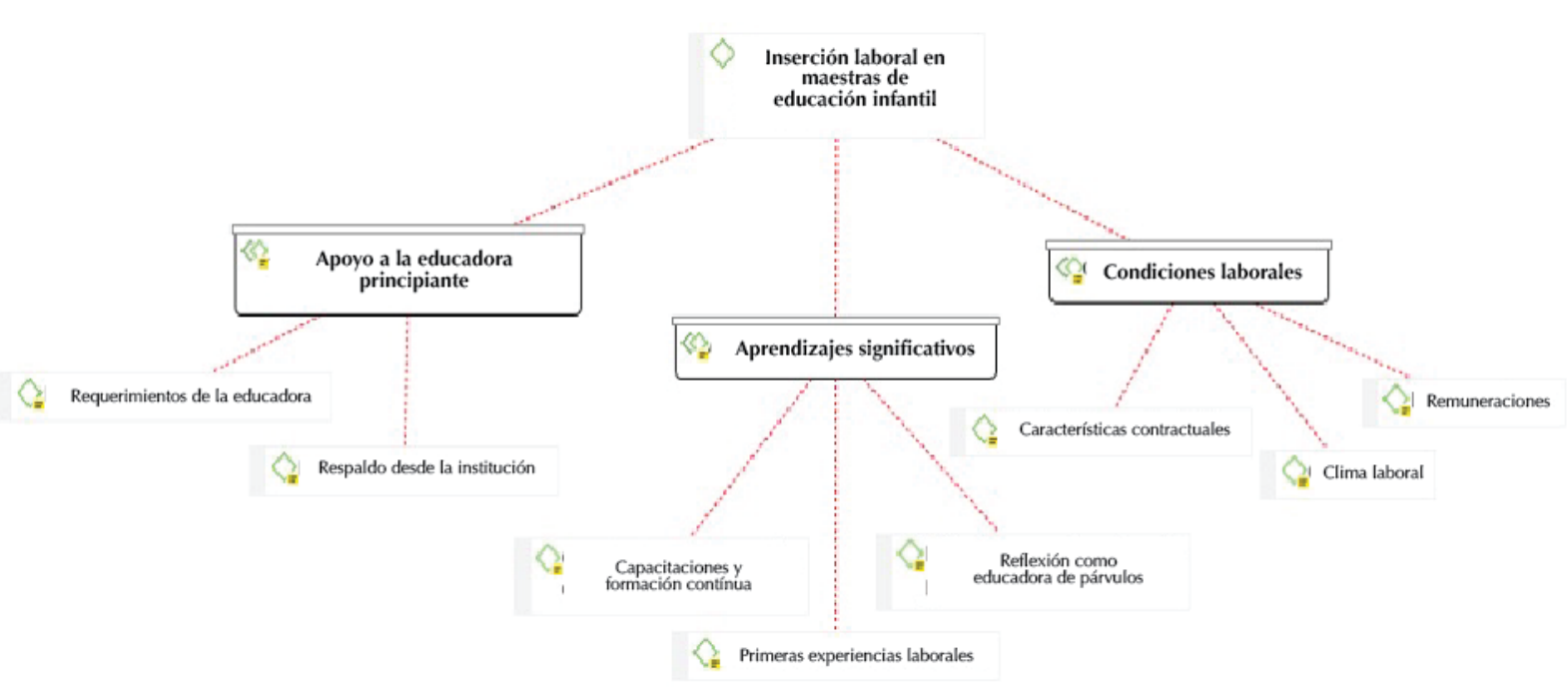

Figura 2. Red semántica entrevistas en profundidad

Fuente: Elaboración propia.

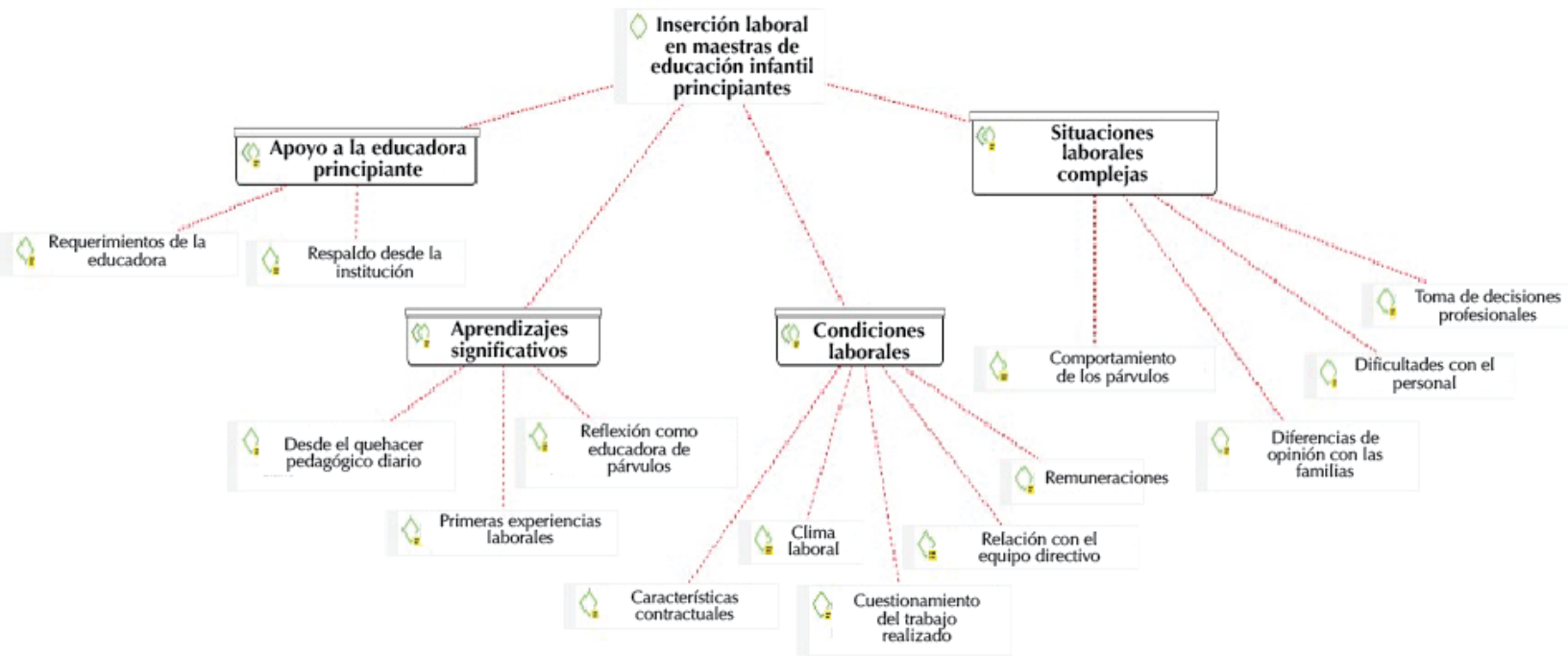

Figura 3. Red semántica grupo focal

Fuente: Elaboración propia.

\section{Apoyo a la educadora principiante}

Se evidencia la necesidad de acompañamiento que demandan las educadoras en sus primeros años de trabajo y de resolver situaciones emergentes que, producto de su inexperiencia, no se sienten capaces de gestionar. El apoyo requerido se relaciona con establecer vínculos con la realidad educativa, lo que le daría mayor confianza y seguridad en su desempeño profesional.

[...] A mí no me sirve que me digan 'ahí están todos los libros' [...] pero qué es lo que me sirve a mí, oriénteme para lo que yo necesito [...] en mi caso, 'ya, necesitamos que tengas tal perfil para cumplir como educadora del nivel [...]' (Entrevista, educadora 3). 
[...] Mi debilidad está en eso del diagnóstico y todas esas cosas [...] de la evaluación, que yo me las he

hecho, porque ahí no hay formato, nada, pero no sé si están realmente bien (Entrevista, educadora 7).

Estas necesidades se relacionan con el poder contar con una educadora con mayor experiencia en el contexto, para orientarlas desde la evaluación de aprendizajes. Se destaca como primordial sentir el apoyo emocional y una mayor empatía por parte del equipo técnico-pedagógico, que se confíe en su labor y se apoyen sus decisiones.

[...] También habían estado en su primer año, entonces era como mucho tirarme como '[...], tienes que hacerlo bien, porque tú ya estuviste en la universidad y tú sabes', pero ¡Pónganse en mi lugar!, ‘Cómo se sentirían ustedes si esto les pasa a ustedes?', 'no, ¡es que es tu culpa!' (Grupo focal, educadora 3).

Se destaca lo significativo del trabajo con pares y de manera interdisciplinaria. Además, se plantea la importancia de las redes de apoyo y la necesidad de que sean más operativas.

Hay trabajo en redes, pero distanciado [...] Le escribo hoy y no tengo respuesta hasta la semana después. [...] He estado con distintos tipos de problemas o de casos de vulneración de derechos, donde he necesitado apoyo [...] y no recibo respuesta hasta en una semana más. Entonces, ¿qué hago? Si todos los días tengo el mismo problema, [...] y ya he intentado todo [...] desde mi rol para ayudar a ese niño [...] (Grupo focal, educadora 2).

\section{Aprendizajes significativos}

Se refiere a la oportunidad que les proporcionan las nuevas situaciones vividas, que permiten importantes aprendizajes, además de darle sentido a su quehacer. El apoyo recibido o no por el equipo directivo y técnico pedagógico y las experiencias laborales vivenciadas en los primeros ańos de trabajo provocan en la educadora cuestionamientos frente a determinadas concepciones. De esta manera, se establecen nuevos aprendizajes y se propicia su desarrollo, tanto personal como profesional, permitiendo el replanteamiento de su quehacer pedagógico.

Porque en el trabajo uno va viendo cosas buenas y cosas malas ... ahí te vas formando, qué es lo que tú quieres hacer [...] Entonces tú misma vas poniendo tu propia identidad, la vas formando a través de las prácticas en el trabajo [...] (Entrevista, educadora 5).

[...] Es como que yo siento que el cambio parte por uno, entonces yo no puedo enseñar sobre buen trato, no puedo enseńar sobre el ser empático con el otro si yo no lo soy, entonces para mí eso ha ayudado [...] este contexto lleno de información mala (Entrevista, educadora 9).

Este aprendizaje se produce en la interacción con diferentes actores del proceso educativo, tales como párvulos, que poseen características propias y diferenciadas. En cuanto a las familias, destacan que lo fundamental es trabajar en conjunto, valorando su rol como primer agente educativo de sus hijos. En relación con el equipo técnico pedagógico, evidencian la importancia de llegar a acuerdos para trabajar en equipo en un ambiente propicio para el aprendizaje

Lo anterior les ha permitido ampliar su mirada con relación a algunas temáticas de su interés, contribuir en su identidad profesional y empoderarse de su rol pedagógico. En este sentido, la reflexión y el significado que se le asigna a lo vivido es determinante, ya que contribuye al mejoramiento de sus prácticas pedagógicas y propicia la reconceptualización de sus marcos de referencias personales, contribuyendo a la construcción de su identidad como profesional de la educación infantil. 
Al comienzo tenía muchas dudas y ansias de poder conocer el tema de diversidad sexual en la infancia, puesto que [...] tenemos muchos prejuicios [...]. Me emocioné [...] al conocer la opinión de nińos, nińas y adolescentes trans [...]. Para mí fue fuerte emocionalmente, cómo de alguna manera nosotros los adultos podemos marcar la vida de un nińo o nińa de manera positiva o negativa [...] (Relatos de vida, educadora 1).

El postítulo terminó de darme la confianza que me faltaba, me puedo enfrentar a la vida con otra postura, y además siento que esa área me gusta mucho y me motiva a seguir estudiando [...], no solo por mí, sino que para darle lo mejor a los niños y niñas [...] Aprendí que uno nunca termina de aprender, y que es muy necesario hacerlo (Relatos de vida, educadora 5).

Dentro de los aprendizajes significativos, destacan las capacitaciones y la formación continua, donde se le atribuye sentido a la adquisición de nuevos aprendizajes, así como las experiencias laborales, visualizadas como una etapa esencial en su desarrollo profesional, formando parte de su crecimiento personal. Por último, mencionan la reflexión como maestra infantil, desde las capacitaciones en las que han participado y las experiencias vividas en su quehacer educativo, comprendiendo que lo personal influye en lo profesional.

\section{Condiciones laborales}

Esta dimensión hace referencia a las características del contexto y elementos vinculados con el trabajo efectuado en la realidad educativa, los cuales se constituyen en sus primeras experiencias en el campo laboral y determinan, dependiendo de las experiencias, la toma de decisiones en la inserción y permanencia laboral. Esta última, principalmente, cuando su contrato tiene plazo de término, como es el caso de las educadoras que realizan reemplazos.

Siento que todo profesional o trabajador busca estabilidad laboral, no reemplazos a corto plazo, es por eso por lo que este contrato trae estabilidad a mi vida, y a la de mi hija, con esto se puede pensar en el futuro, en independizarme (Relatos de vida, educadora 9).

Sumado a lo anterior, el ambiente de trabajo influye en el quehacer pedagógico. Si es positivo, las interacciones comunicativas son favorables y el clima es adecuado para aprender, estableciéndose de esta manera una estrecha relación con el grado de satisfacción laboral-profesional de la educadora

[...]Porque se respeta la opinión del otro, validan tu opinión, son empáticas las compañeras unas con otras (Entrevista, educadora 1).

Por el contrario, si no hay una buena comunicación, se establece un clima poco propicio para el aprendizaje.

[...] que ahora, por ejemplo, con la técnico que tengo no me sigue mucho, se dificulta todo lo que es el trabajo, hay un ambiente un poco tenso que también los niños lo sienten, no hay cercanía, entonces [...] hay un ambiente frío (Entrevista, educadora 4).

Las primeras experiencias, especialmente si se caracterizan por ser negativas, producen cuestionamientos con relación a ser o no una buena educadora.

Cuando entré a mi primer trabajo fue un clima tan malo que yo decía [...] si era buena o no, porque me hacían saber que era mala, entonces nadie confiaba en mí [...], y como era el primer trabajo, estaba incómoda (Grupo focal, educadora 5).

Lo mismo sucede cuando lo solicitado difiere de su pensamiento personal-profesional y de su formación inicial, ocasionando de esta manera, conflictos laborales. 
[...] De repente me cuestiono, 'soy la educadora, soy la que estoy a cargo [...] de su educación y veo que tiene esa necesidad ¿cómo me voy a quedar callada?' respecto de algo que [...] que tiene que trabajarse. Entonces dada la presión, [...] me cuestiono mi carácter [...], yo tengo que preocuparme por la educación del niño, o sea como mi ética, entonces eso me genera conflicto (Grupo focal, educadora 8).

En algunos centros educativos se produce una relación inversa entre la multiplicidad de funciones y los bajos sueldos, y se evidencia un ambiente poco propicio para el aprendizaje al recibir constantes críticas y no tener apoyo del equipo directivo y técnico-pedagógico. Por otro lado, se valora el crecimiento profesional que les permiten algunas instituciones educativas. Sin embargo, en otros centros educativos, el ambiente se caracteriza por extensas jornadas de trabajo, bajas remuneraciones y discriminación, lo que las ha llevado a buscar otras oportunidades laborales.

Recuerdo en una ocasión me dejaron en un jardín donde consideraba que pagaban bien y estaba muy feliz, pero cuando me llamaron para firmar el contrato y llenar mis datos me dijeron que no podía quedarme porque tenía muchos hijos y tenía un bebé muy pequeño. Fue injusto (Relato de vida, educadora 7).

\section{Situaciones laborales complejas}

Estas son situaciones vinculadas principalmente con la dificultad de las interacciones comunicativas con agentes comunitarios. Debido a su falta de experiencia, no cuentan con las competencias necesarias para dar solución de forma oportuna a las situaciones vividas con niños y niñas, familias y equipo técnico-pedagógico.

A mí me pasó con un nińo que tiene trastornos del espectro autista [...] que hubo un momento que fue disruptivo para él en una experiencia, y se desbordó [...], y desde ahí yo me hice una reflexión de '¿Qué hago, cómo lo puedo hacer, cómo me acerco más a él? Tengo que estudiar, tengo que buscar más' [...] (Grupo focal, educadora 1).

[...] Existe como un grado de frustración de repente cuando uno hace todo lo posible porque la familia entienda, comprenda que hay una necesidad educativa especial, [...] él de verdad necesitaba otro tipo de ayuda y la familia se negó [...], y yo igual pienso en ese niño y digo quizás qué realidad está viviendo hoy (Grupo focal, educadora 6).

[...] Nunca alguien me había tratado tan mal. Sucedió el último día del jardín estacional, jamás me habían faltado el respeto de esa forma, fue tanto el mal rato que hasta pensé en retirarme del trabajo a mediodía, ya que me sentía muy incómoda estando en el mismo lugar que esa persona. Me marcó a tal punto que ahora [...] me da miedo decir o pedir cosas a la gente de mi equipo (Relatos de vida, educadora 3).

Los momentos de conflicto que han tenido que enfrentar las educadoras en su quehacer pedagógico les han provocado agotamiento emocional, cuestionamientos y replanteamientos, sentimientos de desilusión y temor.

Otro caso que no me ha dejado lo suficientemente satisfecha me ha enseńado y demostrado que, por mucho que una como profesional ponga todo su empeńo, ganas, estrategias y apoyo, si la familia no logra comprender que una necesidad educativa no limita a un nińo si recibe la ayuda que corresponde [...] uno no puede interferir en sus decisiones familiares [...] (Relatos de vida, educadora 6). 
Esto ha tenido como resultado, en algunos casos, la toma de decisiones vinculadas con la continuidad en la institución educativa. Por otro lado, este tipo de situaciones complejas resultan ser significativas para algunas educadoras, ya que a pesar de las dificultades y de lo difícil de la situación, han podido plantear sus argumentos con claridad y seguridad.

\section{Discusión y conclusiones}

Los hallazgos encontrados favorecen el logro de nuestro objetivo y así contribuir a la caracterización de las complejidades de los primeros años de trabajo de educadoras de párvulos, identificando sus necesidades de apoyo en la construcción de su identidad profesional. En este sentido, se establece que los primeros años como profesional de la educación son claves para su futuro desarrollo, donde la falta de experiencia en aula es una condicionante común que debe ser el foco de atención y eje orientador de las acciones a realizar, tanto por parte de las políticas públicas llevadas a cabo como de los equipos de trabajo de las comunidades educativas a las cuales pertenecen. Estas complejidades son el apoyo a la educadora principiante y sus necesidades, aprendizajes significativos desde lo vivido, ya sea positivo o negativo, y las condiciones y situaciones laborales complejas.

Con relación al apoyo a la educadora principiante y sus necesidades, estas se vinculan con la falta de recursos competenciales en la resolución de situaciones nuevas y desconocidas. Es por esto que el poder contar con las orientaciones de sus pares de mayor experiencia en el contexto y de otros profesionales se transforma en un elemento clave para dar solución a las situaciones emergentes (Robinson, 2019). En este sentido, se puede contemplar un apoyo de mentoría para poder acompañar a los maestros que se inician en la profesión (Gasper \& Walker, 2020).

En lo que respecta a los programas de inducción, en Chile, con la Ley 20.903 de 2016, se propicia el acompańamiento en el primer año a educadoras de párvulos de centros educativos financiados por el Estado. Si bien es un avance en políticas públicas educativas a nivel país, aún existe mucho camino por recorrer para que sea considerado fundamental y parte del proceso formativo de transición de estudiante a educadora, y no solo voluntario para ciertos establecimientos. Si realmente las reformas educativas pretenden mejorar la calidad de la educación con equidad desde el primer nivel educativo, es necesario entregar a todas las educadoras de párvulos las mismas posibilidades de acción y participación.

Sin embargo, más que el acompañamiento desde lo curricular y los requerimientos institucionales, se revela el apoyo emocional y la empatía como elemento articulador para la creación de ambientes propicios para el aprendizaje. Si bien Chang, Kredl y Kingsley (2014) plantean lo importante de desarrollar en la formación inicial lo emocional en la enseńanza, en este estudio se constata la necesidad de continuidad al ejercicio de la profesión, reconociendo así que los primeros ańos de trabajo deben ser vistos como un periodo de transición (Marcelo y Vaillant, 2013), donde el ponerse en el lugar del otro contribuya a la formación y consolidación de equipos técnicos pedagógicos con propósitos claros y acordes con el contexto educativo. De esta manera, las primeras experiencias de trabajo favorecerían la reafirmación del autoconcepto en los educadores (Ávalos y Sevilla, 2010).

Con relación a los aprendizajes significativos, que forman parte de su proceso de formación como educadoras de párvulos, se relacionan con situaciones y experiencias vividas, ya sean positivas o negativas, que le han aportado sentido a su labor pedagógica, contribuyendo a su construcción identitaria. Este aporte solo es efectivo cuando se intenciona en la reflexión de lo vivido.

En este sentido, se establecen como subcategorías comunes las primeras experiencias laborales y la reflexión; la primera se caracteriza por una etapa compleja, cargada de temores, cuestionamientos e inseguridades, dificultades que pueden ser superadas al ir adquiriendo nuevos aprendizajes a través de las situaciones que han tenido que 
enfrentar, por un lado, y por otro, en la interacción con otros agentes educativos y comunitarios. Esta etapa vivida de estudiante a educador es vista por Tejada (2018) como un proceso social que involucra y articula los conocimientos previos de su biografía, la formación inicial y las prácticas de formación llevadas a cabo en diferentes contextos educativos, siendo además un período de grandes desafíos (Loughran, 2014).

En cuanto a la reflexión, esta les permite ampliar su mirada del mundo, lo que les supone un directo beneficio en su desarrollo profesional (Ávalos y Sevilla, 2010; Rojas Torres y Berger, 2017). Dicho de otra manera, el desarrollo profesional de toda maestra en educación infantil es el resultado de la reflexión sistemática (Vergara, 2014), que debe ser intencionada, consciente y en profundidad.

En lo referido a las condiciones laborales, tercera complejidad identificada, sus subcategorías son las características contractuales, vinculadas al tipo de contrato realizado, el clima laboral, con relación al ambiente propicio para el aprendizaje, y las remuneraciones.

Esta categoría se vincula con elementos de la nueva realidad educativa que inciden en su quehacer pedagógico y su continuidad en la institución. En línea con lo anterior, Ávalos (2016) señala que el educador que se inicia cuenta con los aportes entregados por las prácticas formativas de pregrado; por lo tanto, tiene un conocimiento parcial y general de su labor, desconociendo las condiciones laborales de su realidad educativa. La forma en cómo se enfrentan a sus primeras experiencias va en directa relación con las interacciones comunicativas establecidas en el contexto educativo del que forman parte. Esta complejidad concuerda además con lo planteado por Sancho y sus colegas (2014), principalmente en lo referido a la inestabilidad laboral, evidenciándose esto en contratos de reemplazo por periodos cortos o a plazo fijo a uno o dos años.

Del mismo modo, la multiplicidad de funciones que debe realizar transforma su labor pedagógica en esencial y compleja a la vez (Horm, Hyson, \& Winston, 2013). Sin embargo, lo anterior no es considerado por algunas instituciones educativas. Se les exige mucho en una labor que no es socialmente valorada como debería: prueba de ello son las bajas remuneraciones y la falta de reconocimiento social.

En relación con las situaciones laborales complejas vividas con diferentes actores de la comunidad educativa, cuyas subcategorías son comportamiento de los párvulos, dificultades con el personal y diferencias de opinión con las familias, estas provocan en ellas conflictos al no poder resolverlos de manera oportuna. Pillen, Den Brok y Beijaard. (2013) señalan que estas tensiones representan conflictos internos, los que se vinculan con sus sentimientos, percepciones y valores profesionales.

Los hallazgos de este estudio, en cuanto a situaciones laborales complejas, están en directa relación con lo planteado por Solís y sus colegas (2016), al señalar que dentro de las dificultades de los educadores que se inician se establecen las interacciones con los actores de la comunidad educativa, lo que sucede fundamentalmente porque no cuentan con recursos en el manejo de situaciones.

Los primeros trabajos se caracterizan fundamentalmente por la inexperiencia de las educadoras principiantes, condicionados por el contexto, las políticas públicas y sus demandas, y la forma en que la comunidad educativa propicia la integración de este nuevo profesional, donde las dificultades que debe enfrentar son más llevaderas si cuenta con un equipo que oriente y respalde su labor pedagógica. Asimismo, la reflexión crítica que haga de esta etapa de transición va en directa relación con la construcción de su identidad profesional; por lo tanto, los primeros ańos son determinantes en dicho proceso identitario.

El artículo original fue recibido el 6 de enero de 2020

El artículo revisado fue recibido el 12 de agosto de 2020

El artículo fue aceptado el 13 de agosto de 2020 


\section{Referencias}

Arvaja, M. (2016). Building teacher identity through the process of positioning. Teaching and Teacher Education, 59, 392-402. https://doi.org/10.1016/j.tate.2016.07.024

Ávalos, B. (2016). Learning from research on beginning teachers. En J. Loughran \& M. Hamilton (Eds.), International Handbook of teacher education (pp. 487-522). Singapur: Springer. https://doi.org/10.1007/978-981-10-0366-0_13

Ávalos, B. y Sevilla, A. (2010). La construcción de la identidad profesional en los primeros años de docencia: evidencia desde la investigación. Santiago, Chile: Centro de Investigación Avanzada en Educación.

Ávalos, B. y Sotomayor, C. (2012). Cómo ven su identidad los docentes chilenos. Perspectiva Educacional, 51(1), 77-95. https://doi.org/10.4151/07189729-Vol.51-Iss.1-Art.74

Beijaard, D., Meijer, P., \& Verloop, N. (2004). Reconsidering research on teachers's professional identity. Teaching and Teacher Education, 20(2), 107-128. https://doi.org/10.1016/j.tate.2003.07.001

Blanch, S., Comes, P., Edo, M., Estrada, J., Gimeno, X., Morón, M., París, G., y Peire, T. (2017). Cuestionario de autoevaluación de competencias y habilidades personales y prosociales. Bellaterra, España: UAB.

Bolívar, A. (2016). Reconstruir la identidad profesional docente en la sociedad del conocimiento. En J. Valle \& J. Manso (Dirs.), La 'cuestión docente' nuevas perspectivas (pp. 15-29). Madrid, España: Narcea.

Bolívar, A., Fernández, M., y Molina, E. (2005). Investigar la identidad profesional del profesorado: Una triangulación secuencial. F.Q.S: Forum Qualitative Social Research Socialforshung, 6(1), 12. https://doi.org/10.17169/fqs-6.1.516

Bubikova-Moan, J., Naess Hjetland, H., \& Wollscheid, S. (2019). ECE Teachers'views on play-based learning: a systematic review. European Early Childhood Education Research Journal, 27(6), 776-800. https://doi.org/10.1080/1350293X.2019.1678717

Bustingorry, S., Sánchez, I., y Ibáńez, F. (2006). Investigación cualitativa en educación. Hacia la generación de teoría a través del proceso analítico. Estudios Pedagógicos (Valdivia), 22(1), 119-133.

Chang-Kredl, S. \& Kingsley, S. (2014). Identity expectations in early childhood teacher education: Pre- service teachers' memories of prior experiences and reasons for entry into the profession. Teaching and Teacher Education, 43, 27-36. https://doi.org/10.1016/j.tate.2014.05.005

Chase, S. (2015). Investigación narrativa. Multiplicidad de enfoques, perspectivas y voces. En N. Denzin \& Y. Lincoln (Coords.), Manual de investigación cualitativa. Volumen IV. Métodos de recolección y análisis de datos (pp.58-112). Barcelona, España: Gedisa.

Chong, S. (2011). Development of teacher's professional identities: From pre-service to their first year as novice teachers. KEDI Journal of Educational Policy, 8(2), 219-233. Recuperado de https://www.kedi.re.kr/eng/kedi/bbs/ B0000005/view.do?nttId=577\&menuNo=200067\&divisionCd=15\&pageIndex=1

Creswell, J. (2014). Research design. Qualitative, quantitative and mixed methods approaches. Los Ángeles, CA: SAGE.

Correa, J. M., Martínez-Arbelaiz, A., \& Fernández-Plaskoaga, L. (2017). Professional identity and engagement among newly qualified teachers in times of uncertainty. European Early Childhood Education Research Journal, 26(1), 26-36. https://doi.org/10.1080/1350293X.2018.1412013

Escobar-Pérez, J. y Cuervo-Martínez, A. (2008). Validez de contenido y juicio de expertos: una aproximación a su utilización. Avances en Medición, 6(1), 27-36.

Falabella, A., Cortázar, A., Godoy, F., y Romo, F. (2018). Sistemas de aseguramiento de la calidad en educación inicial: lecciones desde la experiencia internacional. Gestión y Política Pública, 27(2), 309-340. https://doi.org/10.29265/gypp.v27i2.469

Gasper, M. \& Walker, R. (2020). Mentoring and Coaching in Early Chilhood Education. Londres, Reino Unido: Bloomsbury Academic.

Horm, D., Hyson, M., \& Winston, P. (2013). Research on early childhood teacher education: Evidence from three domains and recommendations for moving forward. Journal of Early Childhood Teacher Education, 34(1), 95-112. https://doi.org/10.1080/10901027.2013.758541

Huberman, M. (1989). The professional life cycle of teachers. Teachers College Record, 91(1), 31-57. Recuperado de https://psycnet.apa.org/record/1990-05987-001

Ivanova, I. \& Skara-Mincāne, R. (2016). Development of professional identity during teacher's practice. ELSEVIER. Procedia - Social and Behavioral Sciences, 232, 529-536. https://doi.org/10.1016/j.sbspro.2016.10.073 
Ley N 20.903 de Creación del Sistema de Desarrollo Profesional Docente y modifica otras normas. Ministerio de Educación. Santiago, Chile 1 de abril, 2016. Recuperado de https://www.leychile.cl/Navegar?idNorma=1087343

Loughran, J. (2014). Pedagogies of developing teacher. En C. Craig y L. Orland-Barak (Eds.). International Teacher Education: Promising Pedagogies (Part A) (pp.257-272). Londres, Reino Unido: Emerald Group.

Marcelo, C. y Vaillant, D. (2013). Desarrollo profesional docente ¿Cómo se aprende a enseñar? Madrid, España: Narcea.

Martínez, N. (2012). ¿Por qué estudiar la situación del docente novel? En Martínez, N. (Coord.), Alzando el Vuelo: Problemas y modelos de acompañamiento al docente novel (pp. 15-36). Monterrey, México: Fondo Editorial de Nuevo León.

McGillivray, G. (2008). Nannies, nursery nurses and early years professionals: constructions of professional identity in the early years workforce in England. European Early Childhood Education Research Journal, 16(2), 242-254. https://doi.org/10.1080/13502930802141659

Miles, M. B., Huberman, A. M., \& Saldańa, J. (2014). Qualitative Data Analysis. A Methods Sourcebook (3a ed.). Londres, Reino Unido: SAGE.

Ministerio de Educación. (2018). Bases curriculares de la educación parvularia. Santiago, Chile: MINEDUC.

Moloney, M. (2010). Professional identity in early childhood care and education: perspectives of pre-school and infant teachers. Irish Educational Studies, 29(2), 167-187. https://doi.org/10.1080/03323311003779068

Pardo, M. y Alderstein, C. (2016). Estrategia regional de sobre docentes: Estado del arte y criterios orientadores para la elaboración de politicas de formación y desarrollo profesional de docentes de primera infancia en América Latina y el Caribe. Santiago, Chile: OREALC-UNESCO.

Pardo, M. \& Adlerstein, C. (2020). ECEC teachers' paradoxical views on the new Chilean System for Teacher Professional Development. Early Years, 40(4-5), 486-498. https://doi.org/10.1080/09575146.2020.1736008

Pelletier, F. y Morales-Perlaza, A. (2018). Saber e identidad de la profesión docente. De la identidad de estudiante a la de docente principiante. En I. Cantón y M. Tardif (Eds.), Identidad profesional docente (pp. 57-73). Madrid, España: Narcea.

Peeters, J. \& Sharmahd, N. (2014). Professional development for ECEC practitioners with responsibilities for children at risk: which competences and in-service training are needed? European Early Childhood Education Research Journal, 22(3), 412-424. https://doi.org/10.1080/1350293X.2014.912903

Pillen, M., Beijaard, D., \& Den Brok, P. (2013). Tensions in beginning teachers' professional identity development, accompanying feeling and coping strategies. European Journal of Teacher Education, 36(3), 240-260. https://doi.org/10.1080/02619768.2012.696192

Pillen, M., Den Brok, P., \& Beijaard, D. (2013). Profiles and change in beginning teachers' professional identity tensions. Teaching and Teacher Education, 34, 86-97. https://doi.org/10.1016/j.tate.2013.04.003

Perrenoud, P. (2004). Diez nuevas competencias para enseñar. Barcelona, España: Graó.

Robinson, M. S. (2019). Construcción de la identidad profesional de la educadora de párvulos principiante en diferentes centros de educación infantil. Estudio de caso (Tesis doctoral). Universitat Autònoma de Barcelona, Barcelona.

Rojas Torres, M. y Berger, C. (2017). El rol de las experiencias relacionales con profesores en la construcción identitaria de estudiantes de pedagogía. Pensamiento Educativo. Revista de Investigación Educacional Latinoamericana, 54(1), 1-20. Recuperado de https://pensamientoeducativo.uc.cl/index.php/pel/article/view/880

Sancho, J. M., Hernández-Hernández, F., Martínez, S., Padilla Petry, P., Montané López, A., Herraiz García, F.,... \& Losada Iglesias, D. (2014). La construcción de la identidad profesional del profesorado de educación infantil y primaria en la formación inicial y los primeros ańos de trabajo (Identidoc). Síntesis y principales resultados y contribuciones. En J. Sancho, J. Correa, X. Giró, \& L. Fraga (Eds.), Aprender a ser docente en un mundo en cambio (pp. 11-23). Barcelona, Espańa: Universitat de Barcelona.

Solís, M., Núñez, C., Contreras, I., Vásquez N., \& Ritterhaussen Klaunning, S. (2016). Inserción profesional docente: problemas y éxitos de profesores principiantes. Estudios Pedagógicos (Valdivia), 42(2), 331 - 342. https://doi.org/10.4067/S0718-07052016000200019

Stake, R. (2013). Estudios de casos cualitativos. En N. Denzin \& Y. Lincoln (Coords.), Manual de investigación cualitativa. Volumen III. Las estrategias de investigación cualitativa (pp. 428-444). Barcelona, Espańa: Gedisa. 
Tejada, J. (2018). La búsqueda de la identidad laboral del profesorado. En I. Cantón y M. Tardif (Eds.), Identidad profesional docente (pp. 75-93). Madrid, España: Narcea.

Vergara, M. (2014). La identidad de la educadora infantil. Elementos para su comprensión. Pedagogía y Saberes, (41), 111-120. https://doi.org/10.17227/01212494.41pys111.120

Zabalza Beraza, M. A. y Zabalza Cerdeirińa, M. A., (2011). La formación del profesorado en educación infantil. Participación Educativa, 16, 103-113. Recuperado de http://www.educacionyfp.gob.es/dam/jcr:0cf5d740-8cc84fb9-ba44-44d875630059/16_marzo_2011.pdf 